\title{
Translucent whitish papules on the palms after exposure to the water - aquagenic syringeal acrokeratoderma
}

\author{
Magdalena Żychowska, Aleksandra Batycka-Baran, Jacek Szepietowski, Wojciech Baran
}

Department of Dermatology, Venereology and Allergology, Wroclaw Medical University, Wroclaw, Poland

Adv Dermatol Allergol 2017; XXXIV (4): 372-374

DOI: https://doi.org/10.5114/ada.2017.69321

Aquagenic syringeal acrokeratoderma (ASA) was described for the first time in two sisters by English and McCollough in 1996. The authors called this rare entity transient reactive papulotranslucent acrokeratoderma to emphasize the transient nature of translucent papules occurring on the palms after short-time exposure to water and differentiate it from hereditary papulotranslucent acrokeratoderma (HPA), which is characterized by persistent white papules on the palms and soles, hair abnormalities and atopic diathesis [1]. Several synonymous names have been used in the literature to describe this condition, including aquagenic palmoplantar keratoderma [2] and transient aquagenic palmar hyperwrinkling [3].

A 13-year-old boy presented with a 4-month history of burning sensation within both palms evoked by short water contact, which was more pronounced when the water was warm. The sensation was accompanied by the presence of whitish discoloration and translucent papules of the palms. The papules and the burning sensation resolved spontaneously after drying within 15 min. Otherwise, the boy was healthy, did not take any medications and had no atopic background. No other family member had similar symptoms.

Physical examination revealed discrete symmetrical erythema and mild exfoliation on both palms (Figure $1 \mathrm{~A}$ ), while the skin on the soles, hair and nails remained normal. Considering the intriguing medical history, a water immersion test was performed. After 5 -minute contact with water of $20^{\circ} \mathrm{C}$, the boy started to feel burning of the palms, which was accompanied by thickening of the palmar skin and presence of translucent whitish papules and plaques with prominent eccrine pores (Figure 1 B). The burning sensation and translucent papules disappeared after drying within $15 \mathrm{~min}$. A 4-mm punch biopsy was taken from erythematous skin before water exposure and revealed orthokeratotic hyperkeratosis with dilated eccrine ostia and normal dermis (Figure 2). Based on the clinical presentation, positive water exposure test and histopathological findings, the diagnosis of aquagenic syringeal acrokeratoderma was made. The patient was treated with antiperspirant cream containing aluminum chlorohydrate, zinc oxide and urotropine, with a satisfying improvement and no relapse during the follow-up period of 6 months.

Aquagenic syringeal acrokeratoderma is a rare acquired condition characterized by translucent whitish papules and plaques which develop 2-10 min after exposure to water and disappear within 15-60 min after drying. The condition is typically accompanied by itching, tightening and burning sensation, which may be evoked by sweating as well. The palms are the most common location, while the soles were involved in only a few reported cases. Hyperhidrosis is usually present. The condition affects mainly adolescents and young adults (age of onset from 6 to 37 years), with a significant female predominance. The phenomenon is typically symmetrical and develops on both palms simultaneously. In many cases, periods of spontaneous resolution of the condition and good water tolerance have been mentioned [1-5].

The ethiopathogenesis of ASA has not been elucidated yet but the condition is supposed to be the result of impaired permeability of the stratum corneum on the palms, which leads to excessive water absorbance [3].

Typical histopathological findings include orthohyperkeratosis and dilated eccrine ducts [1-6]. The water immersion test should always be performed on physical examination. The typical "hand-in-the-bucket" sign is usually sufficient to establish the diagnosis and biopsy is rarely required.

Aquadynia, aquagenic urticaria and aquagenic pruritus should be taken into consideration in differential diagnosis. Moreover, ASA should be distinguished from physiologic wrinkling as a result of digit pulp vasoconstriction after prolonged water exposure.

The most controversies arise around the nosological position of ASA. English and McCollough postulated that

Address for correspondence: Magdalena Żychowska MD, Department of Dermatology, Venereology and Allergology, Wroclaw Medical University, 1 Chałubińskiego St, 50-368 Wroclaw, Poland, phone: +48 669 422 237, e-mail: magda.zychowska@gmail.com Received: 14.01.2016, accepted: 25.06.2016. 

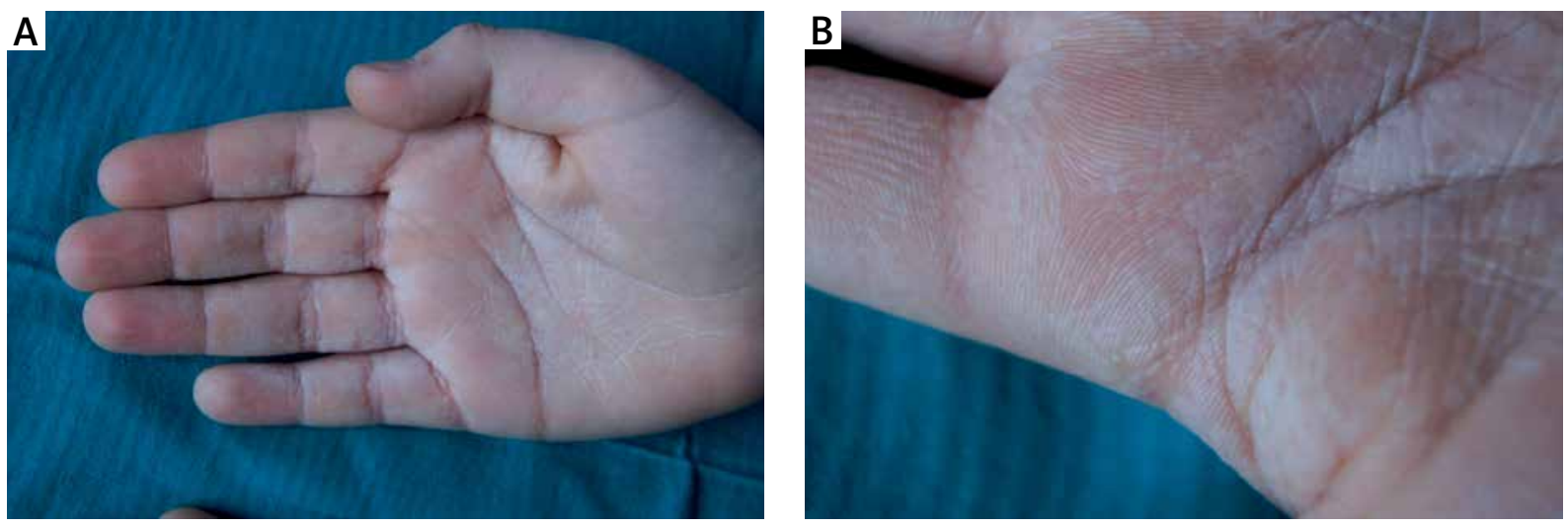

Figure 1. A - Discrete erythema and mild exfoliation on the palm before exposure to water. B - Translucent whitish papules and plaques with prominent eccrine pores after exposure to water

the condition is a variant of HPA [1]. Hereditary papulotranslucent acrokeratoderma is a disorder of an autosomal dominant pattern of inheritance, described in 1973 by Onwukwe et al. [7]. White papules occur in puberty and are concentrated on the margins of palms and soles, which suggests a contributory effect of trauma and pressure. The papules are persistent and there is no association between water exposure and the development of skin lesions. Hereditary papulotranslucent acrokeratoderma is associated with atopic diathesis and hair abnormalities (sparse hair). Histopathology reveals normal eccrine glands with no signs of dilated ostia. Authors of recent papers prefer to separate definitely ASA from HPA. In classic ASA cases, no cutaneous changes persist on the palms between episodes of water exposure. In 2010, Tan and Zhu introduced the term of "atypical ASA", defined by the presence of mild exfoliation or discrete, but persistent, whitish papules on the palms that become more evident after short-time contact with water [6]. Only a few cases of atypical ASA have been described so far. Taking this definition into consideration, the presented patient's condition in another example of an atypical variant of ASA.

Although the condition is characterized by mild course and good prognosis, the burning and tightening sensation may lead to a considerable impairment of life quality. Treatment modalities include aluminum chloride hexahydrate [3], urea with salicylic acid [5] or formalin in alcohol [8]. In cases accompanied by hyperhidrosis, botulinum toxin injections proved to be effective [9].

There were over 30 cases of ASA reported until 2012. In a recent publication in 2015, Erturk-Ozdemir et al. presented a case series of 10 patients, diagnosed with ASA over a short period of 13 months [5]. To the best of our knowledge, this is the first case in a Polish patient. Considering an increasing number of ASA cases described in the literature in the past few years, we presume that the condition is not as rare as it was initially believed. The transient nature of the eruptions may contribute to a large number of undiagnosed patients.

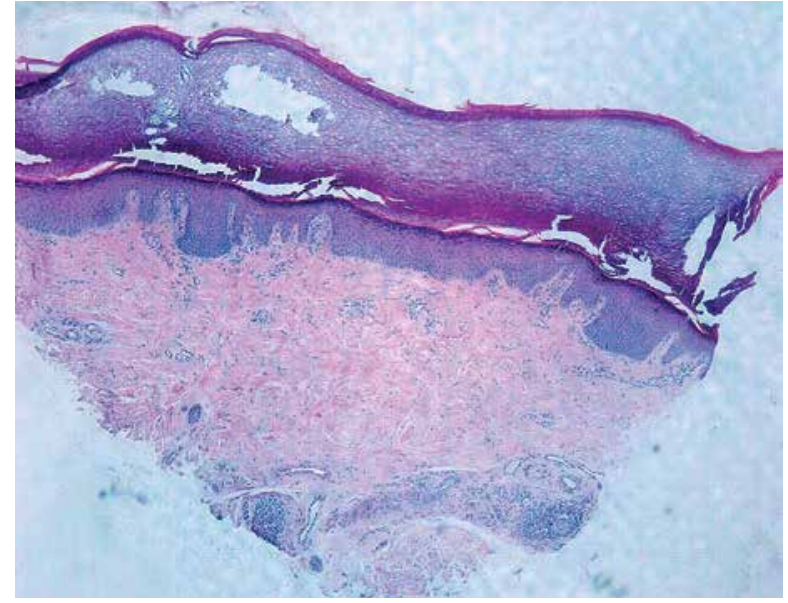

Figure 2. Histopathological findings included orthokeratotic hyperkeratosis with dilated eccrine ostia and normal dermis (10x, hematoxylin and eosin)

\section{Conflict of interest}

The authors declare no conflict of interest.

\section{References}

1. English JC $3^{\text {rd }}$, McCollough ML. Transient reactive papulotranslucent acrokeratoderma. J Am Acad Dermatol 1996; 34: 686-7.

2. Yan AC, Aasi SZ, Alms WJ, et al. Aquagenic palmoplantar keratoderma. J Am Acad Dermatol 2001; 44: 696-9.

3. Neri I, Bianchi F, Patrizi A. Transient aquagenic palmar hyperwrinkling: the first instance reported in a young boy. Pediatr Dermatol 2006; 23: 39-42.

4. Itin PH, Lautenschlager S. Aquagenic syringeal acrokeratoderma (transient reactive papulotranslucent acrokeratoderma). Dermatology 2002; 204: 8-11.

5. Erturk-Ozdemir E, Ozcan D, Seckin D. Acquired aquagenic syringeal acrokeratoderma: a case series of 10 patients. Australas J Dermatol 2015; 56: e43-5.

6. Tan C, Zhu W. Atypical form of transient reactive papulotranslucent acrokeratoderma: a case report and review of the literature. In J Dermatol 2010; 49: 937-40. 
7. Onwukwe MF, Mihm MC Jr, Toda K. Hereditary papulotranslucent acrokeratoderma. A new variant of familial punctate keratoderma? Arch Dermatol 1973; 108: 108-10.

8. Luo DQ, Li Y, Huang YB, et al. Aquagenic syringeal acrokeratoderma in a an adult man: a case report and review of the literature. Clin Exp Dermatol 2009; 34: e907-9.

9. Diba VC, Cormack GC, Burrows NP. Botulinum toxin is helpful in aquagenic palmoplantar keratoderma. Br J Dermatol 2005; 152: 394-5. 